\title{
apterous, a gene required for imaginal disc development in Drosophila encodes a member of the LIM family of developmental regulatory proteins
}

\author{
Barbara Cohen, M. Elaine McGuffin, ${ }^{1}$ Christine Pfeifle, ${ }^{1}$ Daniel Segal, ${ }^{2}$ and Stephen $M$. Cohen ${ }^{1,3}$ \\ ${ }^{1}$ Department of Cell Biology, Howard Hughes Medical Institute, Baylor College of Medicine, Houston, Texas 77030 USA; \\ ${ }^{2}$ Department of Molecular Microbiology and Biotechnology, Faculty of Life Sciences, Tel Aviv University, Tel Aviv, Israel
}

\begin{abstract}
The apterous ( $a p$ ) gene is required for the normal development of the wing and haltere imaginal discs in Drosophila melanogaster. ap encodes a new member of the LIM family of developmental regulatory genes. The deduced amino acid sequence of $a p$ predicts a homeo domain and a cysteine/histidine-rich domain known as the LIM domain. In these domains ap is highly similar to the mec-3 and lin-11 proteins of Caenorhabditis elegans and to the vertebrate insulin enhancer-binding protein isl-1. ap is presumably required for transcriptional regulation of genes involved in wing and haltere development. The nature of the defects in homozygous null mutant flies is consistent with the pattern of $a p$ expression in the larval imaginal discs. ap is also expressed in a complex pattern in the embryo, including portions of the peripheral nervous system (PNS) and central nervous system (CNS). A requirement for ap expression in the larval and adult CNS may be the underlying cause of the defects in hormone production and vitellogenesis described for ap mutations.
\end{abstract}

[Key Words: apterous; LIM domain; homeo domain; transcription factor; imaginal disc; wing development]

Received January 22, 1992; revised version accepted February 25, 1992.

Genetic and molecular analysis of mutations in genes that disrupt development has proved to be invaluable in elucidating the mechanisms by which a normal pattern is generated. Because of the great differences in the lifestyles of the larva and the adult fly, Drosophila organizes different body patterns at different times in its life cycle. The segmentation and dorsoventral patterning systems organize the larval body pattern (for review, see St. Johnston and Nüsslein-Volhard 1992). In addition, the primordia of the adult body, known as imaginal discs, are laid down in the embryo. Some aspects of patterning in the disc occur concomitant with specification of the discs in the embryo. For example, the subdivision of the disc primordium into anterior and posterior compartments is imposed on the nascent disc/Wieschaus and Gehring 1976; Simcox et al. 1989).

Several genes known for their roles in pattern formation in the Drosophila embryo have also been shown to play important roles in the normal development of the imaginal discs (e.g., Kornberg 1981; Spencer et al. 1982; Baker 1988; Phillips et al. 1990). The segment polarity genes wingless, engrailed, and patched were first identified by virtue of viable mutant alleles that produced wing defects (Sharma and Chopra 1976; Ecker 1929,

${ }^{3}$ Corresponding author. cited in Kornberg 1981; Phillips et al. 1990). wingless and decapentaplegic encode secreted intercellular signaling molecules that are required for normal growth and patterning of all imaginal discs (Padgett et al. 1987; van den Heuvel et al. 1989; Gonzalez et al. 1991). Other segment polarity genes, such as patched, have been implicated in the receptor side of these signaling processes (Phillips et al. 1990; Ingham et al. 1991). It is probable that the functions of the intercellular signaling genes in patterning of both the embryonic segment and the imaginal discs are similar (for review, see Whittle 1990; Wilkins and Gubb 1991).

Many additional genes, for which no embryonic function is known, are required for growth and pattern formation of the imaginal discs. A number of genes of this type have been identified in screens for late larval or pupal lethals (Shearn et al. 1971; Shearn and Garen 1974; Kiss et al. 1976). One particularly interesting class of genes is that required for the development of a subset of imaginal discs (for review, see Shearn 1978). A number of genes that are required for development of the wing have been identified, including apterous (ap), vestigial (vg), and defective dorsal discs (ddd) (Butterworth and King 1965; Fristrom 1969; Simcox et al. 1987).

ddd gene function is needed during larval stages to promote growth of the entire wing and haltere discs. 
Like wingless, $d d d$ functions in a nonautonomous manner, suggesting a secreted product (Simcox et al. 1987). vg encodes a nuclear protein that is expressed throughout the disc and that is required in the wing and haltere blade (Williams et al. 1991). In the absence of $v g$ function, extensive cell death is observed in the region of the wing blade, although the body wall structures that derive from the disc develop normally (Fristrom 1969; Bownes and Roberts 1981).

In this report we present the molecular cloning of the ap gene. ap encodes a member of the newly described LIM family of developmental regulatory proteins. These proteins contain both a sequence-specific DNA-binding motif, the homeo domain, and a cysteine/histidine-rich domain. $a p$ is expressed in the wing and haltere discs during larval development and is required in a cell-autonomous manner for the development of the wing and haltere structures. ap is also expressed in a subset of cells in the embryonic and larval central and peripheral nervous systems (CNS and PNS, respectively). We suggest that other aspects of the ap phenotype may depend on its expression in the adult brain.

\section{Results}

Identification and cloning of a P element inserted in the ap gene

An insertional mutation disrupting the ap locus was identified in a screen of enhancer detector P-element lines. Flies homozygous for a P-element insertion (known as rK568) exhibit a wingless and haltereless phenotype characteristic of the ap mutation. The P-element insert maps to the cytogenetic locus $41 \mathrm{~F}-42 \mathrm{Al}, 2$ idata not shown). The insertion behaves as a mutant allele of $a p$ in crosses to deletions that uncover the ap locus and to other alleles of $a p$. We designate this insertional mutant $a p^{K 568}$. The location of the insertionally mutated gene was genetically mapped with respect to a series of overlapping deletions (Fig. 1). The affected gene lies in the interval defined by the overlap between the left breakpoints of $D f(2 R) n a p^{12}$ and $D f(2 R) n a p^{9-2}$, corresponding to the cytogenetic interval $41 F 8-42 \mathrm{~A} 1$. This interval has been shown to contain the ap gene ( $R$. Kreber, pers. comm.) but differs from a previously published cytogenetic map location of the gene (41B-C; Lindsley and Zimm 1985).

DNA flanking the P-element insert was recovered by plasmid rescue and used to isolate genomic DNA from the ap locus (Fig. 1). Sequences within the 2.2-kb EcoRI fragment of phage aplC (where the element resides) hybridize to embryos and to imaginal discs in a pattern identical to that visualized by $\beta$-galactosidase activity in

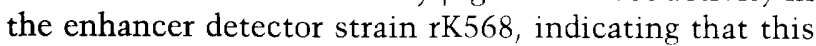
fragment contains transcribed sequences. The $2.2-\mathrm{kb}$ EcoRI fragment was used to isolate cDNA clones from a Drosophila imaginal disc cDNA library. The structure of the $>21-\mathrm{kb}$ ap transcription unit is shown in Figure 1. ap cDNA clones fall into two size classes corresponding to estimated transcript sizes of 3.2 and $4.3 \mathrm{~kb}$. Mapping and sequence analyses suggest that the two transcript classes differ only in the length of their $3^{\prime}$-untranslated tails.

\section{Isolation of an ap lack-of-function mutant}

Most previously described ap mutations are homozygous viable. Flies mutant for strong ap alleles eclose normally but lack virtually all wing and haltere structures (Fig. 2; Butterworth and King 1965; Wilson 1981a; L. Sarov and D. Segal, in prep.). The P-element insertion rK568 produces a strong ap phenotype when homozygous (not shownl. Although they are severely affected phenotypically, homozygous rK568 mutant animals are able to express the ap transcript (data not shown; for details, see Materials and methods). Consequently, it is possible that rK568 retains some $a p^{+}$function. In the hope of isolating a definitive lack-of-function mutation in the gene, we produced a small deletion in the ap gene by imprecise excision of the rK568 P-element insertion (Fig. 3).

A total of 175 strains carrying independent excision events were analyzed genetically. Of these, $55 \%$ showed complete phenotypic reversion of the insertional mutation to produce wild-type flies. Molecular analysis of one such revertant (UGO5) showed that the P-element had been excised from the gene (Fig. 3). Within the limits of resolution of the Southern blot, UGO5 appears to be a precise excision. This result demonstrates that the P-element insertion was the cause of the ap mutation in enhancer detector stain rK568.

An additional $45 \%$ of the strains were homozygous viable and continued to show ap mutant phenotypes after excision of the resident $P$ element. Half of these exhibited a strong phenotype, similar to that of the parental insertional mutant. Molecular analysis showed that one of the strong mutants (UGO35) was caused by an imprecise excision of the $\mathrm{P}$ element resulting in a deletion of $\sim 6 \mathrm{~kb}$, which removes the first exon of the ap transcription unit (Fig. 3). Double-labeling experiments using the longest ap cDNA clone show that homozygous UGO35 animals do not express the ap transcript in the embryo, in the larval brain, or in the imaginal discs /data not shown). If alternate forms of the transcript exist, they cannot share a significant amount of coding sequence with the major transcript forms. The severity of the mutant phenotype in UGO35 homozygotes is not distinguishable from that of UGO35 in trans to a deletion for the locus. UGO35 homozygous individuals therefore define the null phenotype of the ap locus (Fig. 2).

Other excision strains exhibited less severe wing phenotypes than that caused by the original insertion. The phenotypes of these alleles are characteristic of other hypomorphic alleles of ap described previously (Butterworth and King 1965; Wilson 1981a; L. Sarov and D. Segal, in prep.). The production of hypomorphic alleles by excision of $\mathrm{P}$ elements has been documented previously (e.g., Searles et al. 1986). These investigators 
A

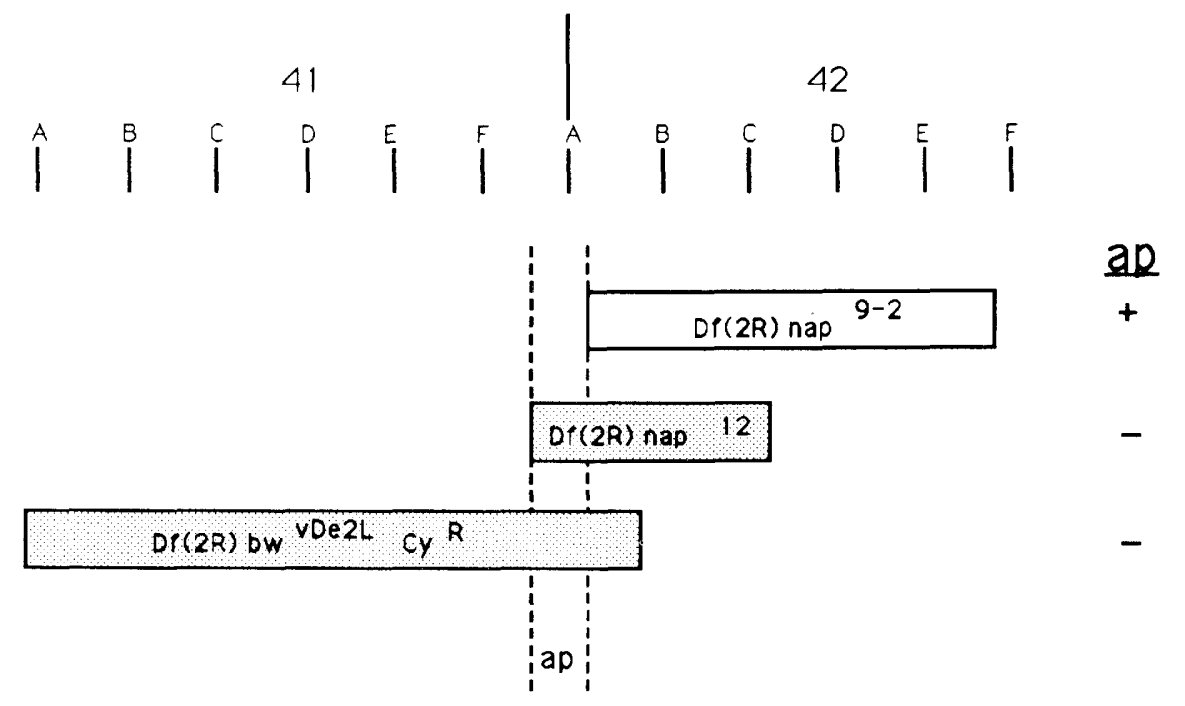

B

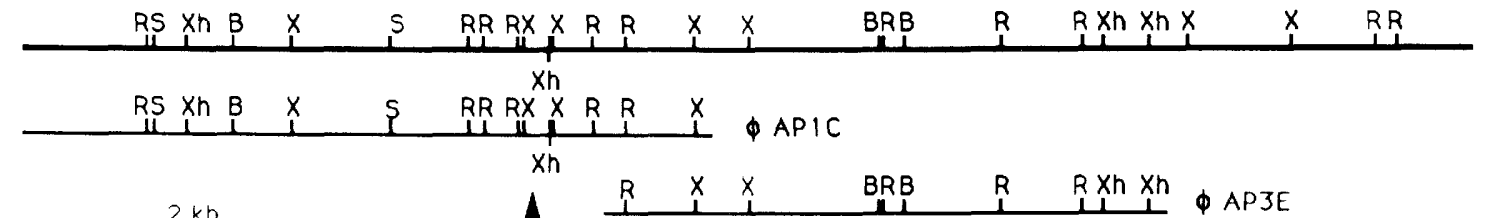
$\underline{2 k b}$

C

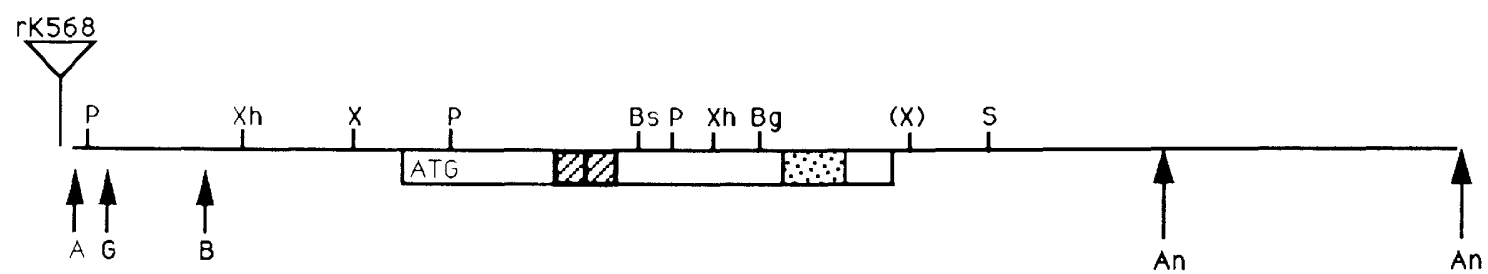

$\underline{200 \mathrm{bp}}$

Figure 1. Molecular organization of the ap locus. (A) The cytogenetic localization of the ap gene is represented with respect to chromosomal deletions that delimit the locus. ap is contained within deletions $D f(2 R)$ nap $p^{12}$ (cytology: 41F5-8; 42Cl-3) and $D f(2 R$ ) $b w^{v D e 2 L} C y^{R}$ (cytology: $\left.41 \mathrm{~A}_{;} 42 \mathrm{~A} 7,8\right)$. The region is delimited on its telomeric side by $D f(2 R)$ nap ${ }^{9-2}$ (cytology: 42A1,2; 42E5-7), which does not contain $a p .(B)$ Physical map of genomic DNA containing the ap locus. Three genomic phages cover the region that contains all of the ap exons found in the cDNA clones. The 1-kb XbaI fragment of $\phi A P 1 C$ indicated by the broken arrow contains the genomic DNA recovered by plasmid rescue of the P-element insertion rK568. The approximate positions of ap-coding sequences are depicted as boxes below the map. Exon positions were determined by hybridization with cDNA probes. $(C)$ Physical map of $a p$ cDNA clones. The location of the rK568 insertion was determined by DNA sequencing to be 42 bp upstream from the $5^{\prime}$ end of the longest cDNA clone. The 5' ends of cDNA clones A, B, and G are indicated by arrows below the map. The coding region is depicted by an open box. Coding sequences begin in the first exon. The positions of the LIM domain (hatched boxes) and the homeo domain (stippled box) are indicated. Polyadenylation sites observed in cDNA clones are shown (An). Restriction mapping indicates that the majority of cDNA clones recovered start in the region indicated by A, B, and G. Assuming that there is only one transcription start site, transcripts of 3.2 and $4.3 \mathrm{~kb}$ are predicted. (B) BamHI; (Bg) BglII; (Bs) BssHII; (P) PstI; (R) EcoRI; (S) SmaI; (X) XbaI; (Xh) XhoI. 


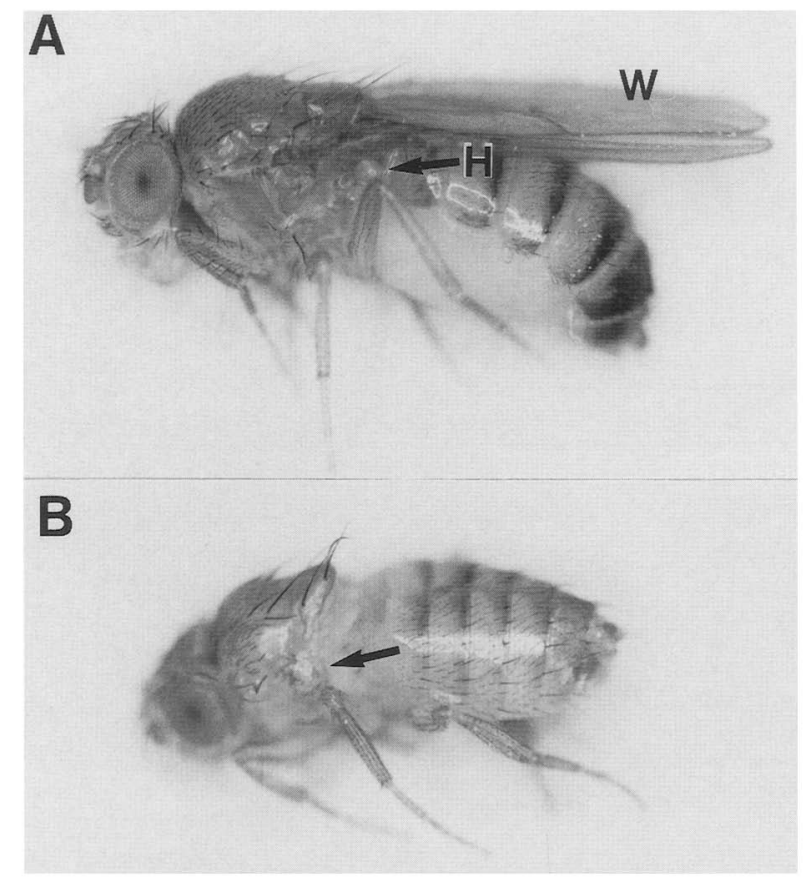

Figure 2. Morphological defects in ap mutants. $|A|$ Side view of a wild-type fly, showing the normal appearance of the wing $|W|$, haltere $(\mathrm{H})$, and the pattern of bristles on the dorsal thorax. $|B|$ Comparable view of an ap null mutant fly of genotype ap Ucio35; $a p^{U G O 3.5}$. The wing is reduced to a small stump (arrow). The haltere is similarly reduced (not visible in the picture). A number of large bristles (machrochaetae) are missing from the thorax near the wing, and this region appears reduced in size relative to the rest of the thorax. These flies are short lived and females are nonvitellogenic.

showed that the severity of the mutant phenotype caused by an insertion was reduced by internal deletions within the resident $\mathrm{P}$ element. It is possible that the hypomorphic alleles recovered in our excision screen represent this class of imprecise excision event.

\section{ap expression in the imaginal discs}

ap mRNA is expressed throughout the regions of the imaginal discs that give rise to the dorsal surface of the wing and haltere blades and at lower levels in the regions of the wing and haltere discs that form the dorsal thoracic body wall structures, the notum and scutellum (Fig. 4). ap expression is highest in the region of the wing hinge. In mild ap mutants the hinge region is defective, leading to flies with essentially normal wings, which are held out from the body in an unusual posture (Wilson 1981a). Increasingly severe mutants cause reductions in the wing and haltere, and in the strong mutants these structures are absent, but the notum and scutellum are essentially normal. The P-insertion allele rK568 exhibits this strong hypomorphic phenotype. The null mutant allele UGO35 produces a slightly more severe defect, in which particular dorsal thoracic bristles are absent. Absence of thoracic bristles has been observed previously in some strong alleles of ap (Butterworth and King 1965), suggesting that these may also be null alleles. Thus, there is a good correlation between the pattern of $a p$ expression and the range of defects observed in wing development in ap mutants. Regions that express high levels of the gene product are most sensitive to reductions in gene activity.

$a p$ is also expressed in the presumptive fourth tarsal segment of the leg imaginal discs and in a central spot in the antennal portion of the eye antenna disc. The leg and antenna appear to develop normally in the ap null mutant, suggesting no essential role for the gene in morphogenesis of these structures. However, some ap alleles have been found to produce unusual kinking of the legs (L. Sarov and D. Segal, in prep.). The significance of this

Figure 3. Isolation of ap lack-of-function mutants. $|A|$ A genetic scheme for the isolation of ap mutants is shown. Mutagenesis results as an indirect consequence of imprecise excision of the P-element insertion in $a p$. The scheme is based on genetic selection for excision events, which are identified by elimination of a dominant marker $\left(r y^{+}\right)$, carried on the P-element insertion. Step 1 combines the rK568 insertion (chromosome II) with a stable source of P-element transposase located on chromosome III (Robertson et al. 1988). Males carrying the transposase (dominantly marked with $S b$ ) and carrying the P element (i.e., those not dominantly marked $S p$ ) were mass mated with females carrying a dominantly marked $\left|r y{ }^{\dagger}\right|$ second chromosome in a ry mutant background. The excision events represented by single males lacking a copy of the $r y^{-}$gene were amplified in step 3 , and stock carrying the putative mutation was established by sister-brother mating at step 4 . The progeny of these crosses were scored for the production of $a p$ mutant offspring. $(B)$ Genomic Southern blot showing the molecular nature of selected excision events. rK568/CyO denotes DNA taken from flies carrying the insertion line and heterozygous for the balancer chromosome CyO. UGO35/CyO is DNA from a balanced excision strain. UGO35 and UGO5 are DNA from flies homozygous for independent excision events derived from the rK568 chromosome. DNA in the first set of four lanes was digested with EcoRI. DNA in the second set of four lanes was digested with Xbal. Different hybridizations of the same filter are shown. (Left) Hybridized with a 1.4-kb XbaI fragment (indicated by the vertically striped box in C). The excision of the P element in UGO5 is apparently precise, as indicated by the restoration of the $1-\mathrm{kb} \mathrm{XbaI}$ fragment that contained the rK568 insert (arrow, left). The excision in UGO35 removed $6 \mathrm{~kb}$ of DNA flanking the insertion, including the first exon of the $a p$ transcription unit. $\mathrm{XbaI}$ fragments of 4 and $1.4 \mathrm{~kb}$ are deleted in the UGO35 excision (cf. lanes UGO35 and UGO5), and the 1-kb XbaI fragment that contained the element is replaced by a small ( $\sim 400 \mathrm{bp}$ ) fragment (not visible with the probes shown here). (Right) Probed with a subfragment from the right-hand side of the adjacent EcoRI fragment. (This fragment, indicated by the cross-hatched box, shows a number of polymorphic EcoRI sites.) DNA from this fragment is present in the UGO35 excision. Note the identical size of the nonpolymorphic $\mathrm{XbaI}$ fragments in all four lanes. $(C)$ Physical map depicting XbaI and EcoRI restriction sites in the rK568 P-element insertion and the result of the UGO35 excision. (Solid boxes) ap exons 1 and 2 ; (stippled box) the UGO35 deletion. Sites in parentheses are polymorphic between cDNA and genomic DNA that derive from different strains. 
A

1) introduce transposase to rK568 stock:

\section{2) mass mate $S b S p^{+} F 1$ males:}

$$
\frac{S p}{C y O} ; \frac{r y^{506} S b P[r y+\Delta p-3](99 B)}{T M G, U b x}
$$$$
X \underset{\text { Cyo, P[ry } \left.{ }^{+}\right](w g / l a c z)}{\frac{P\left[l a c z, r y^{+}\right](r k 568)}{r y}} \frac{r y^{506}}{+}
$$

$\frac{\left.\text { Pliacz, ry }{ }^{+}\right](r K 568)}{C y O} ; \frac{r y^{506} S b P[r y+\Delta 2-3](99 B)}{r y}$

$X \frac{\left.\text { lethal (2) P[ry }{ }^{+}\right] \text {b Adh cn }}{c y 0} ; \frac{r y^{506}}{r y}{ }_{+}^{506}+$

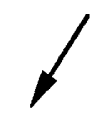

3) select single ry- males:

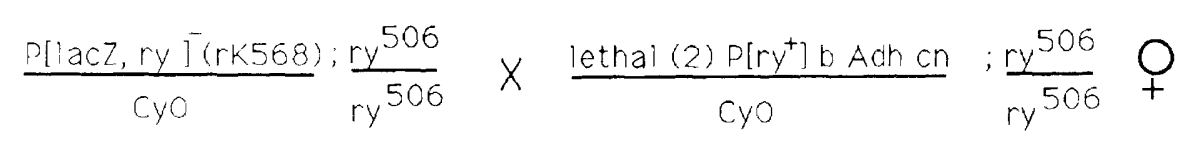

4) establish stock:

$$
\frac{P[\mathrm{lacz}, \mathrm{ry}](\mathrm{rk568})}{\text { Cyo }} ; \frac{r y^{506}}{r y^{506}}
$$

B

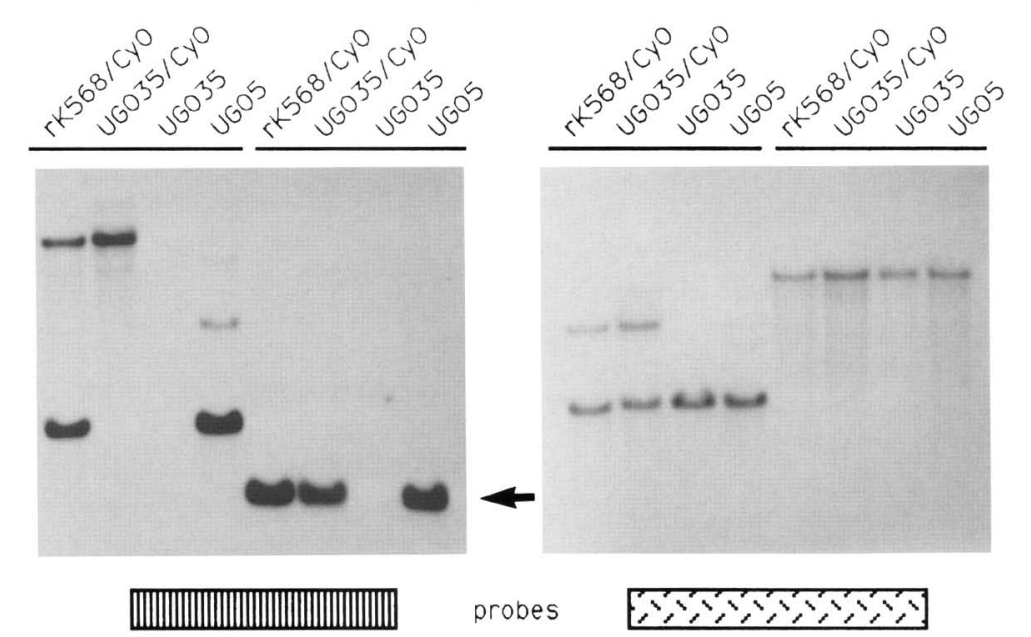

C

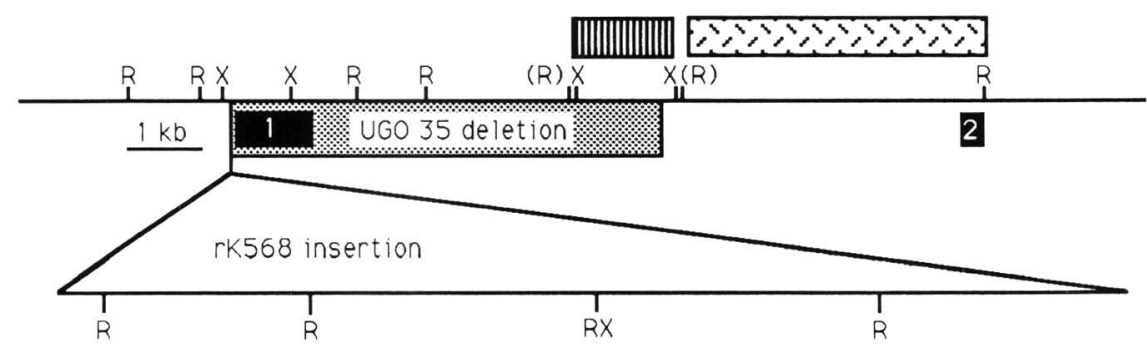

Figure 3. (See facing page for legend.) 
Cohen et al.

phenotype is unclear, because the null mutant produces no defect.

ap expression in the embryo

The pattern of ap expression during embryogenesis is complex and dynamic. Earliest ap expression is seen during germ band extension in the mandibular lobe of the embryonic head (Fig. 5A,B). These cells flank the invaginating stomodeum in the germ band-extended embryo and invaginate as the floor of the pharynx during germ band retraction. The role of ap expression in this tissue is unclear because ap mutant larvae obviously feed successfully. $a p$ is also expressed from germ band extension until the end of embryogenesis in the cells that form the anus.

By the end of germ band extension ap is expressed in segmentally repeated dorsolateral and ventrolateral groups of cells (Fig. 5C,D). By the completion of germ band retraction the ap-expressing cells have invaginated beneath the ectoderm (Fig. 5E,F,J). Double-labeling experiments show that most of the ap-expressing cells in these clusters are associated with the developing PNS (Fig. 6). ap-expressing cells are closely associated with, but clearly distinct from, the PNS neurons, visualized by monoclonal antibody $22 \mathrm{Cl}$. The locations of the apexpressing cells suggest that some of them may be accessory cells associated with a subset of both chordotonal and external sensory organs (Ghysen et al. 1986; Bodmer and Jan 1987).

Clearly, other cells in the thoracic segments cannot be accessory cells of the PNS. In flattened preparations, some of the ap-expressing cells are very elongated and lie below the PNS. On the basis of their morphology and position, it is possible that these additional ap-expressing cells may be larval muscle precursors (Bate et al. 1991). In particular, the group of ap-expressing cells that lie dorsal to the leg primordia in the thoracic segments correspond closely in position to a group of identified muscle precursor cells. Although the function of the ap gene product in the PNS and the muscles is not known, ap expression in these cells is clearly not essential for viability of the larva. Changes in the fate of a substantial portion of the PNS as a result of mutations in genes of the achaete-scute locus have been shown previously to be compatible with survival to adulthood (DamblyChaudiere and Ghysen 1987).

$a p$ is also expressed in the embryonic and larval CNS. ap expression in the presumptive embryonic brain begins in the fully germ band-extended embryo and persists through embryogenesis (Fig. 5C,E,F,I). During germ band retraction, ap begins to be expressed in the ventral nerve cord. Expression is initially in one cell per hemisegment (Fig. 5G). Additional cells soon begin to express ap, increasing the number to two to four cells per hemisegment (Fig. 5H). This pattern of embryonic nervous system expression may be related to, or may be the precursor of, the later expression of $a p$ in the larval CNS (Fig. 4).
A possible neuroendocrine origin for the pleiotropic defects in ap mutants

In addition to the morphological defects in wing disc development, ap mutants exhibit a number of physiological defects: Females are sterile, and mutant flies fail to undergo larval fat body histolysis and are short-lived (Butterworth and King 1965). Differential regulation of ap expression in a variety of tissues and at different times in development may explain the complex array of defects observed in ap mutants. Genetic characterization of a large number of $a p$ alleles has shown that defects in wing/haltere development can be functionally distinguished from defects in other processes, for example, female fertility (Butterworth and King 1965; Wilson 1981a,b; L. Sarov and D. Segal, in prep.).

Other manifestations of the ap phenotype may reflect indirect consequences of ap function in the brain. A1though ap mutant females are nonvitellogenic and fail to undergo larval fat body histolysis (Butterworth and King 1965; Postlethwait and Weiser 1973), ap expression cannot be detected in the female germ line or in the fat body by histochemical staining for $\beta$-galactosidase activity in rK568 flies or by RNA in situ hybridization (data not shown). The defect in vitellogenesis is not an autonomous property of the ovary, because ap mutant ovaries can become vitellogenic after transplantation into a normal host (Postlethwait and Handler 1978). Both phenotypes are associated with reduced levels of juvenile hormone (JH) production (Altartz et al. 1991), and topical application of a $\mathrm{JH}$ analog to the female abdomen restores vitellogenesis and, to some extent, fat body histolysis (Postlethwait and Weiser 1973).

$\mathrm{JH}$ secretion from the corpus allatum is reduced in $a p$ mutants (Altartz et al. 1991). However, it is unlikely that ap has a direct role in regulating $\mathrm{JH}$ production or secretion, because $a p$ is not detectably expressed in the corpus allatum (not shown). ap is, however, expressed in the larval (Fig. 3) and adult brain (not shown). Corpus allatum function is believed to depend on neurotrophic signals from the brain, suggesting that the defects in hormone production /and secondarily, in vitellogenesis and fat body histolysis) are an indirect result of ap function in the brain (Altartz et al. 1991). However, on the basis of mosaic analysis, Wilson (1981b) suggests that the defect leading to abnormal $\mathrm{JH}$ secretion may lie in the abdomen.

One other striking phenotype of ap mutant flies is premature death, within a few days of eclosion. It has been suggested, on the basis of genetic mosaic analysis, that early death reflects a defect in Malpighian tubule function (equivalent to the kidney) or in the hindgut (Wilson $1981 \mathrm{~b}$ ). Histochemical staining for $\beta$-galactosidase activity in rK568 flies shows that ap is not detectably ex pressed in the Malpighian tubules.

\section{ap encodes a predicted transcription factor}

The diverse array of ap mutant phenotypes may be explained by the observation that $a p$ encodes a predicted 


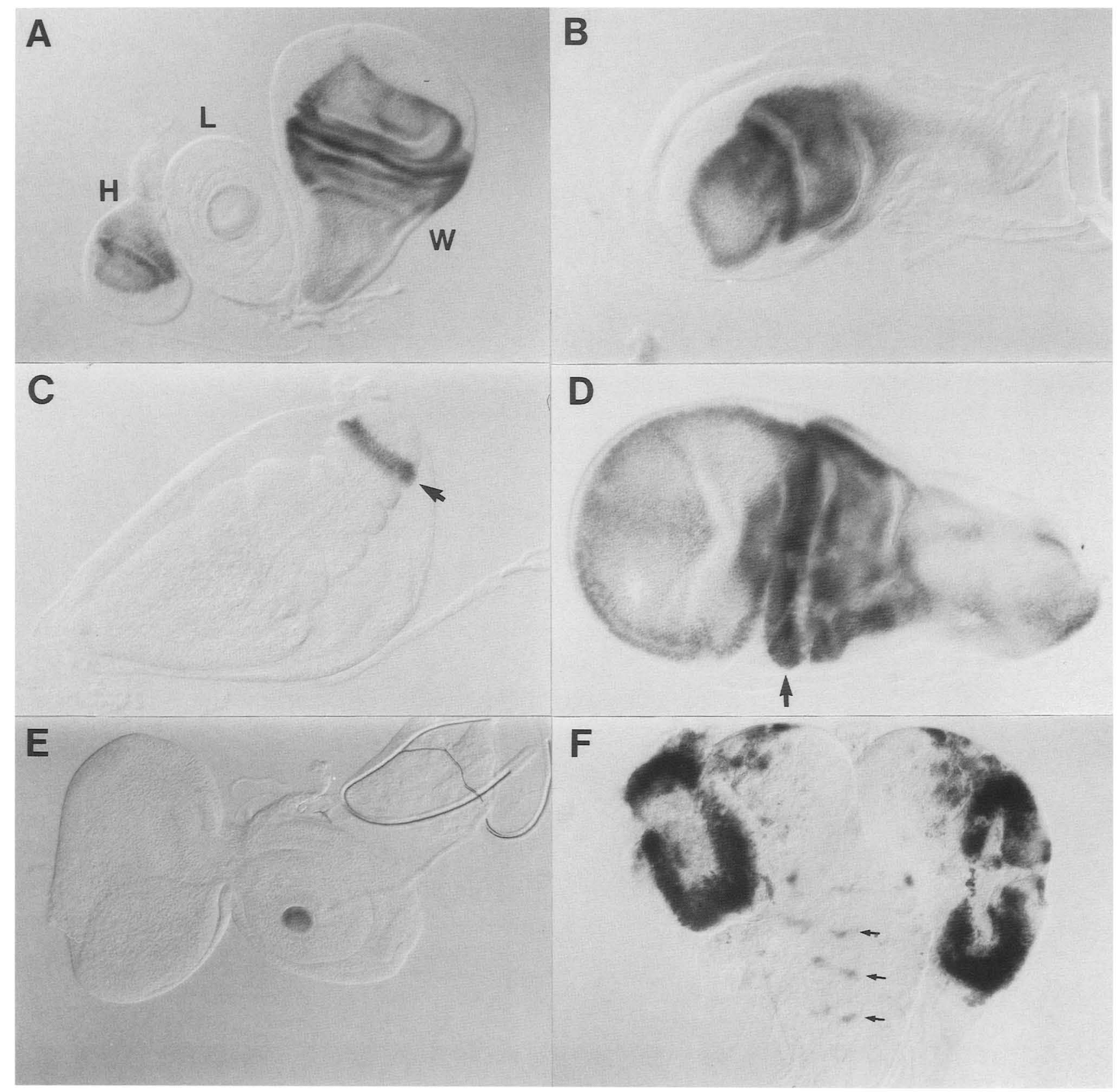

Figure 4. ap expression in larval and pupal imaginal discs and brain. The pattern of ap expression in the imaginal discs and the larval brain is visualized by histochemical staining for $\beta$-galactosidase activity in the insertion line rK568. The pattern visualized in this way is indistinguishable from the pattern of $a p$ transcript expression in these tissues (not shown). The morphology of the samples is better preserved in the histochemical staining procedure. $(A)$ Shown are leg $(\mathrm{L})$, wing $(\mathrm{W})$, and haltere $(\mathrm{H})$ discs from a mature third-instar larva. The dorsal surface of the wing and haltere blades are labeled, as well as a ring in the tarsal segment of the leg. Note the elevated levels of expression in the hinge region and around the wing and haltere margins, compared with the center of the wing blade and capitellum. $(B)$ Prepupal haltere disc beginning to undergo eversion. $(C)$ Prepupal leg disc beginning to undergo eversion. ap is expressed in the fourth tarsal segment (arrow). (D) Prepupal wing disc undergoing eversion. Note that the label covers the entire dorsal surface of the disc. Expression is strongest in the hinge region (arrow). The difference in the appearance of labeling between the third-instar and prepupal discs reflects the reorganization of the disc during eversion (see Bryant 1978). (E) Eye-antennal disc from a third-instar larva showing ap expression in the disc center. $(F)$ The complex pattern of ap expression in the brain. Note also the repeated clusters of cells in the ventral nerve cord (arrows).

protein that is likely to regulate transcription (Fig. 7). ap encodes a new member of the LIM family of develop- mental regulatory proteins (Way and Chalfie 1988; Freyd et al. 1990; Karlsson et al. 1990). Proteins in this family 


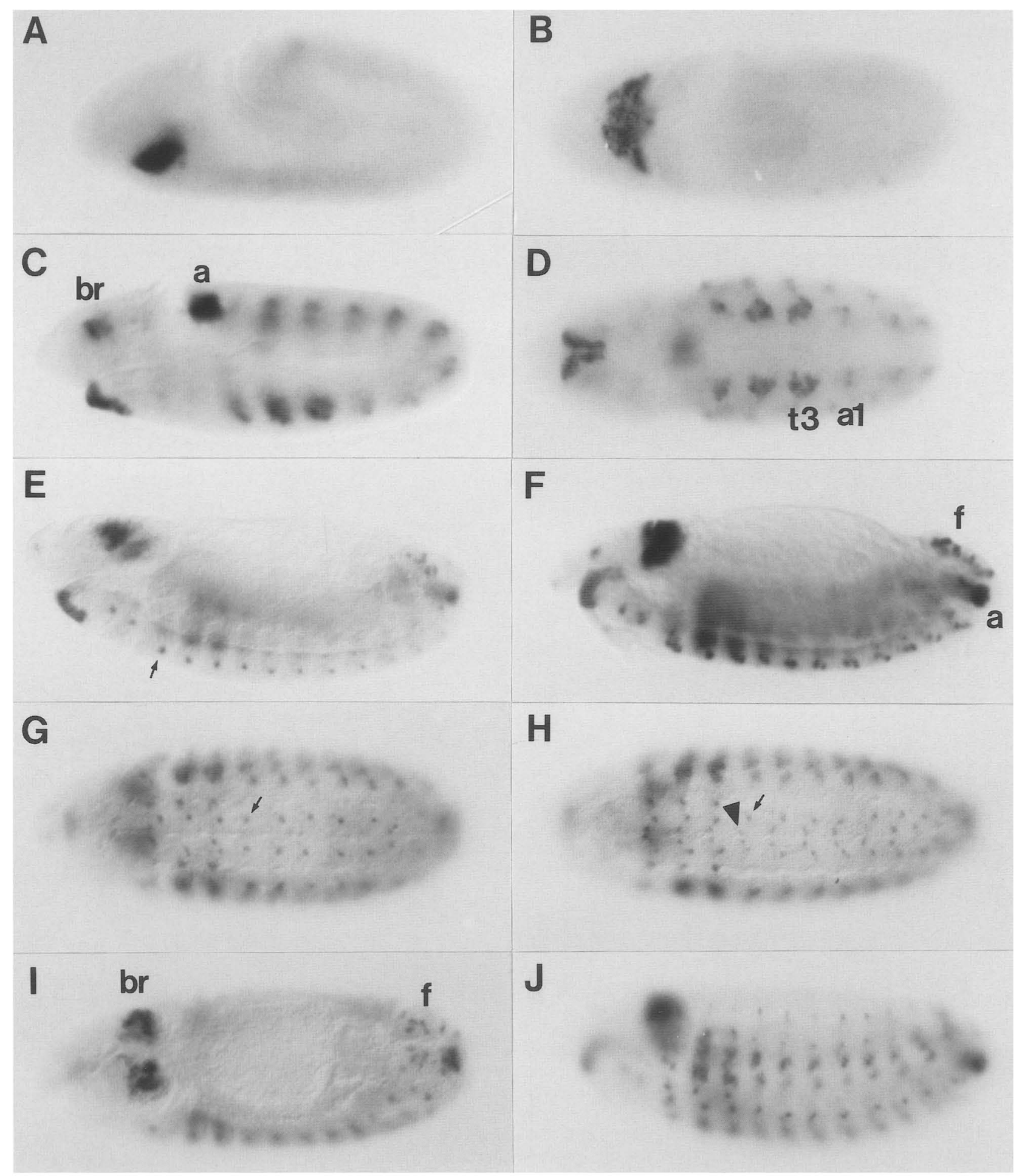

Figure 5. ap expression in the embryo. The pattern of ap transcript expression was visualized by whole-mount in situ hybridization (Tautz and Pfeifle 1989|. ap is first expressed during germ band extension in the mandibular lobe of the embryonic head. $\mid A$, lateral view; $B$, ventral view). By the completion of germ band extension, additional domains of expression arise in the presumptive brain (br), in the region of the hindgut (probably the presumptive anus, based on later expression $(a)$, and in segmentally repeated pattern $\langle C$, lateral view). The ventral view in $D$ emphasizes the difference in the pattern of expression in the thoracic (t3) and abdominal (al) segments. $\mathrm{t} 2$ and $\mathrm{t} 3$ express $a p$ in more cells than are seen in al. $E$ and $G$ show the early pattern of expression in the CNS, initially one cell per hemisegment in the abdomen [(arrow in $E$ (parasaggital view); and in $G$ (ventral view)]. An additional cell per hemisegment is labeled in thoracic segments. $F$ and $H$ show slightly older embryos in which additional CNS cells are labeled (arrowhead). Note also the expression in the filzkörper $(\mathrm{f})$ and anus $(\mathrm{a})$, as well as in the brain (br). I shows a dorsolateral view to highlight expression in the brain and filzkörper. / shows a surface view of the PNS and other cells expressing ap. All of these cells lie below the ectodermal layer. 


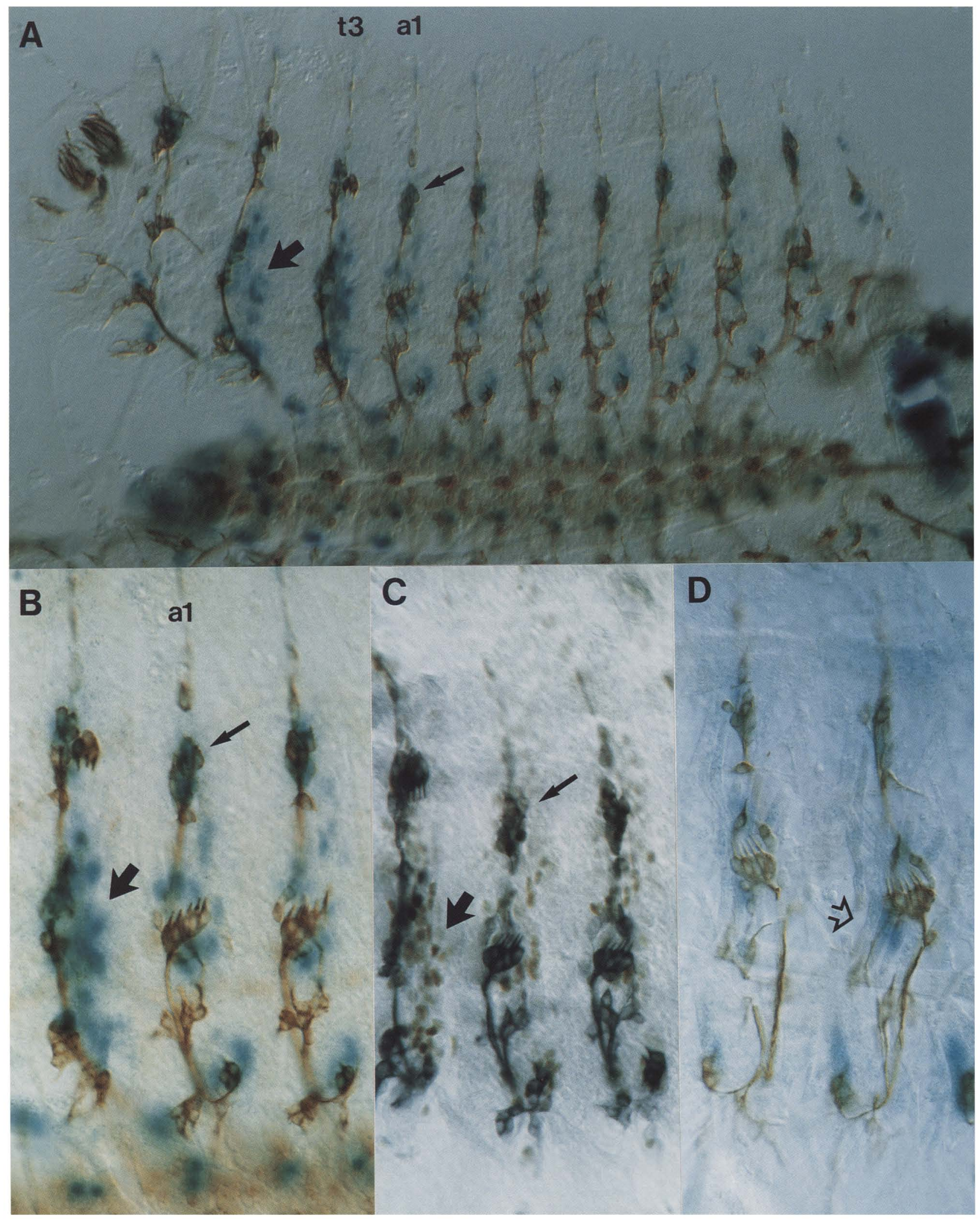

Figure 6. (See following page for legend.) 
are characterized by the presence of a conserved cysteine/histidine-rich motif known as the LIM domain and by the presence of a distinctive class of homeo domain. The LIM proteins are also generally similar in overall organization, except that the ap protein has a prolinerich domain between the LIM domains and the homeo domain (Fig. 8). The presence of a proline-rich domain has been correlated with a transcriptional activation function in other proteins (Mitchell and Tiian 1989). The predicted ap protein shows extensive sequence similarity to the lin-11, isl-1 and mec-3 proteins in both the LIM domain and in the homeo domain. ap, lin-11, and isl-1 are slightly more similar to one another in both domains than any of them are to mec-3.

\section{Discussion}

$a p$ is a new member of the LIM family of developmental regulatory proteins. The LIM family was initially defined by the genes $\operatorname{lin}-11$, isl-1, and mec-3 /Way and Chalfie 1988; Freyd et al. 1990; Karlsson et al. 19901. On the basis of the homeo domain, these genes are believed to function as regulators of transcription. Consistent with this suggestion, both the mec- 3 and is $1-1$ proteins have been shown to bind DNA in a sequence-specific manner (Karlsson et al. 1990; M. Chalfie, pers. comm.l. The cysteine/histidine-rich LIM domain has recently been shown to bind both zinc and iron (Li et al. 1991). A number of alternative proposals have been put forth concerning the functional role of this domain, including redoxsensitive protein-nucleic acid interaction, direct protein-protein interaction, or a structural role (Freyd et al. 1990; Li et al. 1991).

The Caenorhabditis LIM genes function in the specification and/or maintenance of cell fate. $l i n-11$ is required for one daughter of an asymmetric cell division in vulval development to adopt a cell fate different than that of its sister cell (Ferguson et al. 1987). mec-3 is required, in conjunction with the homeo domain protcin unc-86 and at least one other gene, for the specification of touch receptors (Way et al. 1988; Chalfic and Au 1989|. mec-3 appears to be activated in response to the initial signal specifying these cells and acts to maintain the determined state through autoregulation /Way and Chalfie 1989; Way et al. 1991). It is intriguing that the ap gene is associated with some mechanosensory elements of the developing PNS, and with some muscle precursors in the Drosophila embryo. It will be interesting to determine whether ap expression plays a role in the specification or maintenance of cell fate in these cells. The pattern of 22C10 labeling does not reveal gross changes in the organization of PNS neurons in the ap null mutant (data not shown). A more detailed analysis will be required to ask whether subtle alterations can be detected in the developmental fates of accessory cells, in which ap is expressed.

\section{What is the role of ap in wing development?}

Using a temperature-sensitive allele of ap, Wilson (1981a) has shown that ap function is required during the second and third larval instars for normal wing development. Taking advantage of molecular markers to identify mutant larvae, we find that development of the wing disc is already morphologically abnormal early in the third larval instar (data not shown). These observations suggest that $a p$ is an important early regulator of wing development. Consistent with the identification of the ap gene product as a presumed transcriptional regulatory protein, ap function in the wing is cell autonomous in genetic mosaics (Wilson 1981b). By analogy to lin-11 and mec-3, it is possible that ap may regulate the expression of genes required for either growth or pattern formation in the developing wing and haltere discs.

ap is only one of several genes in which mutations lead to loss of the wing and haltere structures but that leave the dorsal thorax relatively unaffected. Mutations in the $v g$ gene cause defects in wing and haltere development similar to those described for ap (Fristrom 1969; Bownes and Roberts 1981; Williams et al. 1991). As in ap null mutants, the wing and haltere discs are reduced in size in the third-instar larva in strong vg mutants (Fristrom 1969; Williams et al. 1991). The wingless phenotypes of both ap and $v g$ are associated with extensive cell death in the presumptive wing blade region of the wing discs (Fristrom 1969). ap and $v g$ are expressed in broad overlapping domains of the wing disc. $a p$ is expressed throughout the dorsal surface of the disc, including the presumptive body wall; $v g$ is expressed at high levels on both dorsal and ventral surfaces of the wing blade and at lower levels in the presumptive body wall /Williams et al. 1991). Thus, the domains of $a p$ and $v g$ expression

Figure 6. A subset of PNS and muscle precursor cells express ap. (A) Flattened preparation of an embryo, double-labeled to visualize cells expressing $a p$ (blue, $\beta$-galactosidase activity) and PNS neurons (brown, monoclonal antibody $22 \mathrm{C} 10$ ). The pattern of $a p$ expression visualized by the enhancer detector insertion is indistinguishable from that visualized by in situ hybridization to ap transcript. $(B)$ Detail of $\mathrm{t} 3$ and al segments in A. Most ap-expressing cells in the abdominal segments are closely associated with PNS neurons (small arrow). Some of these are likely to be accessory cells. In the thoracic segments, additional cells, which cannot be PNS accessory cells, are labeled (large arrows). (C) Double label showing ap-expressing cells labeled with antibody to $\beta$-galactosidase (brown) and with $22 \mathrm{C} 10$ to label PNS neurons (gray). In contrast to the X-gal staining, the antibody shows predominantly nuclear localization of the $\beta$-galactosidase protein. It is clear from these preparations that some of the cells expressing ap cannot be PNS cells (e.g., large arrow). Note, however, that because of the stability of the $\beta$-galactosidase protein, the pattern of expression may reflect, to some extent, the history of $a p$ expression in the cells. $(D)$ Labeled as in $A$ and $B . \beta$-Galactosidase activity staining shows that some ap-expressing cells are very elongated (open arrow). Some of these cells lie below the PNS clusters and may be stretch receptors or muscles associated with the chordotonal organs. On the basis of their location, other such cells are more likely to be muscle precursors. 
Pstl

1 GACGGCTGGTTGTCCTCACGATTTTAAGTAGATCAGCTGCAGACCAAAGTCGGTAGTCGAGTTTCGAATTTAAGTCGGTTTTTGTTTTTGGAAGCGGTGCGCGTATTCGATTTTTTGTTTT 121 GACTTGAGCTCGGCTGTAGTTTCGGTTTGCCAGAACTGGATCGAAGGAGAATCGACACAGAAACAGGGAGTTGGCGAAACGGATACAAAACTGAAGTTGGATCGGTAATCGCCTGCCCGG 241 TCATAAACAACAACACAAGGGAGCACAGCCGCATACGCATACGCGCCTCTGTCTCTAATGCCTTATCAGTAACAGGCATCGTCGAGAGTCGTAGGAAGAGCCCGACAAAGGCCGACCTTT CAP-B

361 ACCACTCAATTTGGATCTGATGTGCCATTCTCGCGCTGTCTCGTAAATTAATGTCATAAATTAACTTTAGATTTCTTACGCTAACCGGTGGTCGTCAGCGGTCCGAAAACGCCTTTTGCGC 481 TCGAGATACAGGGGCGGTCCCTAATCAATACAAATATCCTACCTGCCGCCGAGTCAAGTGTTTTAGTGAGTGTGTGACCCTATGTTCCTTATATTACCTTGGTGCTAAGGAGAAAGTTTA 601 AAAAAAGCGGCATTCAAATCGTAGTTTGTGCAATAAGAAAAGGCAAGCCAATGGTGTCAATCTCTAAGACAACGCCGAAGTGATGCATGTCCGCAAAGTAATAGATCAAGTTGTGCGCAC 721 ACTTATTTCCGGCGAGCCTAGCCGGGCATCTAATCGTGGCCAGTGTCAAGTGTCCAATAGCACATCTAGCCGGGTCTAGAGGATTACCCAAGACCAACGTAGCACCGCAGCCCTTTTAA 841 GGAGGCATAAGGCTCGCAAGACACAACGCACAGCCTACACAACGACAAACATAACACGACCGACGGACATCATCCTTGGAAATAGACCTCGTGTCATTCCAAAAGACGCAC ATG GGC 1) Met Gly

958 GTC TGC ACC GAG GAG COC CCT GTG ATG CAT TSG CAG CAG AGC GCA AGA TTT CTT GGG CCC GGC GCA AGG GAG AAA AGT CCA ACA CCA CCT

3) Val Cys Thr Glu Glu Arg Pro Val Met His Trp Gln Gin Ser Ala Arg phe Leu Gly Pro Gly Ala Arg Glu Lys Ser Pro Thr Pro Pro Psti

1048 GTA GCA CAT CAA GGG AGC AAT CAA TGT GCC AGT GCT GCA GGT GCA AAT AAC AAT CAC CCA TTG TTC CGC GCG TEC TCC TCA TCC TCG TGT 33 Val Ala His Gin Gly Ser Asn Gin Cys Gly Ser Ala Ala Gly Ala Asn Asn Asn H1s Pro Leu Phe Arg Ala Cys Ser Ser Ser Ser Cys 1138 CCA GAT ATT TGT GAT CAT AGT ACA AAG CCA TTT GGC AAC GCT TAC GOC ACC GAG TCA TIT AGA AGC TAC GAA ACA GCC GAT CGT GCT ACC 63. Pro Asp Ile Cys Asp H1s Ser Thr Lys Pro Phe Gly Asn Ala Tyr Gly Thr Glu Ser Phe Arg Ser Tyr Glu Thr Ala Asp Arg Ala Thr 1228 TTT GAG GAC TCA GCC GCC AAA TTC TCC ATC AGC CGC AGT CGA ACA GAC TGC ACG GAG GTC AGC GAC GAG ACG ACG TCG GCC ATA TCA TTC 931 Phe Glu Asp Ser Ala Ala Lys Phe Ser Ile Ser Arg Ser Arg Thr Asp Cys Thr Glu Val Ser Asp Glu Thr Thr Ser Gly Ile Ser Phe

1318 AAG ACC GAA CCC TTC GGA CCG CCC AGC AGT CCC GAG TCC ACA AGC GAT AGC AAA ATA ACG CGC AAC CTC GAC GAC TGC TCC GQC TGC GGA 123 Lys Thr Glu Pro phe Gly Pro pro Ser Ser Pro Glu Ser Thr Ser Asp Ser Lys Ile Thr Arg Asn Leu Asp Asp Cys Ser Gly Cys Gly 1408 CGT CAG ATA CAG GAT CGC TTC TAC CTC TCC GCT GTG GAA AAA CGG TGG CAT GCA AGT TOC CTA CAG TOC TAC GCC TGT CGG CAG COG CTG 153 Arg Gin Ile Gin Asp Arg Phe Tyr Leu Ser Ala Val Glu Lys Arg Trp His Ala Ser Cys Leu Gin Cys Tyr Ala Cys Arg Gln Pro Leu LIM 2

1498 GAA CGG GAA TCC TCA TOC TAC TCA CGT GAC GGC AAC ATT TAT TGC AAA AAC GAT TAT TAT AGT TTT TTT GGT ACT CGC CGA TGC TCG CGC 183 Glu Arg Glu Ser Ser Cys Tyr Ser Arg Asp Gly Asn Ile Tyr Cys Lys Asn Asp Tyr Tyr Ser Phe Phe Gly Thr Arg Arg Cys Ser Arg BosHII

1588 TGC CTG GCC TCC ATC AGC TCC AAC GAG CTG GTC ATG GGC GCC AGA AAT CTT GTT TTT CAC GTC AAC TGC TTC TGC TGC ACT GTC TGC CAC 213 Cys Leu Ala Ser Ile Ser Ser Asn Glu Leu Val Met Arg Ala Arg Asn leu Val phe His Val Asn Cys Phe Cys Cys Thr Val Cys His Pstl

1678 ACG CCA CTG ACA AAG GGA GAC CAG TAC GGC ATC ATC GAC GCC CTC ATC TAC TGC AGG ACC CAC TAC AGC ATA GCC AGG GAG GGG GAT ACC 243 Thr Pro Leu Thr Lys Gly Asp Gln Tyr Gly Ile Ile Asp Ala Leu Ile Tyr Cys Arg Thr His Tyr Ser Ile Ala Arg Glu Gly Asp Thr Xhol

1768 GCC TCA TCC AGT ATG AGC GCC ACC TAC CCG TAC AGC GCC CAG TTC GGC TCA CCC CAC AAC GAC TCC TCG AGC CCG CAC TCG GAC CCT AGT 273 Ala Ser Ser Ser Met Ser Ala Thr Tyr Pro Tyr Ser Ala Gin Phe Gly Ser Pro His Asn Asp Ser Ser Ser Pro His Ser Asp Pro Ser 1858 CGG AGC ATT GTT CCT ACG GGC ATC TTT GTG CCC GCG TCC CAC GTA ATC AAC GGA CTG CCG CAG CCA GCT CGT CAA AAG GGC AGG CCC CGC 303 Arg Ser Ile Val Pro Thr Gly Ile phe Val Pro Ala Ser His Val Ile Asn Gly Leu Pro Gln Pro Ala Arg Gln Lys Gly Arg Pro Arg Bglll

1948 AAG CGC AAG CCC AAG GAC ATC GAG GCG TTC ACC GCT AAC ATA GAT CTC AAC ACT GAG TAC GTG GAC TTT GGC CGA GGC TCG CAC CTA AGC 333 Lys Arg Lys Pro lys Asp Ile Glu Ala Phe Thr Ala Asn Ile Asp Leu Asn Thr Glu Tyr Val Asp Phe Gly Arg Gly Ser H1s Leu Ser homeodomain

2038 TCC TCG TCG CGC ACC AAA CGA ATG CGA ACC TCG TTT AAG CAT CAC CAG CTG CGT ACC ATG AAG TCC TAC TIT GCC ATT AAT CAT AAC CCT 363 Ser Ser Ser Arg Thr Lys Arg Met Arg Thr Ser Phe Lys His His Gln Leu Arg Thr Met Lys Ser Tyr Phe Ala Ile Asn His Asn Pro 2128 GAT GCA AAG GAT CTA AAA CAA ITG TCA CAG AAA ACT GGT ITA SCA AAG AGG GTC CTA CAG GTC TGG TTT CAA AAT GCA AGG GCC AAA TGG 393 Asp Ala Lys Asp Leu Lys Gln Leu Ser Gln Lys Thr Gly Leu Pro Lys Arg Val Leu Gln Val Trp Phe Gln Asn Ala Arg Ala Lys Trp 2218 CGC CGC ATG ATG ATG AAG CAG GAT GGC AGC GQC CTG CTG GAG AAG GGC GAA GGC GCT CTG GAC CTC GAC AGC ATC TOC GTG CAC AGT CCT 423 Arg Arg Met Met Met Lys Gln Asp Gly Ser Gly Leu Leu Glu Lys Gly Glu Gly Ala Leu Asp Leu Asp Ser Ile Ser Val His Ser Pro 2308 ACG TCG TTT ATA TTG AGA GGA CCG AAC AGC ACA CCG CCA CTT AAC TTG GAC TAA CGGATGCTCAGGAAACGCGAAAACAAAATGATAGTGACAAAGGAAATG 453 Thr Ser Phe Ile Leu Arg Gly Pro Asn Ser Thr Pro Pro Leu Asn Leu Asp ...

2410 GGCGCAGCAGTCACCTCCGACATCATACGTTCTTATGCCAGTTGTCTATTCGCGTGCCTCATACACAGTCCCGGACCCGGCTTGACTACTGAATCCGGATACTTGTCTAGATCAAGCACG

2530 GCTAGCTCTTGAATGCTATGGGCTGCCTTGTCGGATGGGAATCAAT'TTGGAAAGCTTGTCTTGTGTTCAAAATCGAAAAATTGATTCATCCACACGTTAAACGCCCGGGAAACGCCTCGC 2650 AAAATGCTAAGAAAATATGTATTTTTGTATTTGCCTATTGACCGTTTCCAGCACTACTCGCCTCGTCCTTGCCCCACATGTTGTTTCTATAGTTTTAACGTTCACTTGTAAATATGTACT 2770 GTAATCACATAGTTTATTTCAAACGCATCCATATTAGCCTGACTCCCCTGTTTTTTGTATTCTTTTAAAGCGCAGAATATCAATTCGAACATTGAAATTATGAATCAAAAGAAGGTGGAT 2890 TCATGCAAAGATTACCAACTTGTAACGTTCTTTCTAAAACTGTCATAATAATTAAGAAAACACAACTTCGTTAAATTTCAATTTGTATAGAAAATAGTTATGTTACTATACCAGTCAAAA 3010 TTTTTAAACATCAAACATGCAGAATTTACGAATTAAAGTATGACTAAAACTTTTAAATACCAAACAGCGAAACGTGTGATATTTTAAATTTGTTTTTTTCTTTTTCAGTGACACGACTA 3130 AAAGATACCCGTTACATTGTAAAATAATCTTGCCAATATAGATGCGGCCAGGTCATGCCCTTCCTCATATGAATTGCCCGCTGGCAATGTTTTTACCAAGCTTTCACCTTCCGATTAGGT 3250 TTGGTGGTCATTTAAGTAAATTCCGATTTAAACTTTGTTTTTAATACGGGAGCGTGTCACTTCCTTTCGGTATAAAAACATAGGTGAGGAAAAGTTTGAGGCCAACCTTACGGCGCACCA 3370 AGCGGCACACAAATGAAATGAAATTCTTTAGCTTCTATAGTAGTCGAAAAACGATCCTTATCAAGCATAAATGCTTATATCTCCTGTAACATTCTTTTTGACCAGTCTAGTTTACACGTA 3490 TGTTCTACGAGGAACGGACCGATTGTCCTATGAGAGCTATAGGTTTTACCATATGTATATGCATGCATTACTTCAGCTCCATCTCTTTTTGATTCATAAACGTATAATGGTTTGAGAGTA 3610 AACATTTTGTGGCGTATATAGTTCAAGTTTACATATTTAGAGTACGGTGCCCAAGACGCGCTTTAAAAATATTAAAACGGGTTGTTAATGAAGTCCCATATGTATAATTGTCTAGGTACA 3730 TGGATATTGTTAATATATTATTATTTTTATTTAAAGAGCTCTCAACAATATGTATGAAGAAATCTGCGTATATCAACATATAATGAAATGCTGATGCATTCGTATTATATATATGTAACT 3850 AATATTTATTGCCCAAATTTAATAGTTAGAGACGAATTTAGTTTAGAACAGACTATACAATAAAGTCCATATGTACATGTAGTATTACGGTAAAGTAATAGCTTCGCTCAAGCATACGAC 3970 CAGGTTCGTAAGCTATTAATACGAAACTGTAATTATAATCAAATCAGTAAACACTGGAAGATTTGTACTTACAAATAGTTAACTCATAAGTCTTACCATAGAAGCTTAAATAAAACGTAT 4090 TCAAGCAAA

Figure 7. Nucleotide sequence and deduced amino acid sequence of the ap protein. The 4098-bp sequence of the longest class of cDNA clones is presented. It was compiled from the complete sequence of cDNA clone cAP-B, with the extended $5^{\prime}$-untranslated region of cAP-A added. The start of CAP-B at residue 365 is labeled. The long open reading frame encoding the deduced $a p$ protein begins at residue 952. A second in-frame ATG codon occurs at amino acid residue 11 . The sequence context of the first ATG codon [GCACATG] is a reasonable match for the Drosophila consensus translation start site [CAACATG] (Cavener 1987). An in-frame stop codon is located $60 \mathrm{bp}$ upstream from the first ATG. The deduced amino acid sequence predicts a protein of 469 amino acids containing two LIM domains (sequence in italics, labeled LIM 1 and LIM 2) and a homeo domain (underlined). The positions of restriction sites shown on the map of the cDNA in Figure 1 are labeled. The locations of poly(A) tail addition in the $3^{\prime}$-untranslated region are shown by solid diamonds, and consensus polyadenylation sequences (AATAAA) are underlined. 
Figure 8. Comparison of $a p$ with mec3, lin-11, and isl-1. (A) Sequence comparison of the LIM domains of ap, lin-11, mec-3, isl-1, and TCTP proteins. The conserved cysteine and histidine residues of the two LIM repeats are aligned. Amino acid residues conserved among ap, lin-11, mec-3, and isl-1 are highlighted in bold and shown above the $a p$ sequence. Amino acid residues identical to those in $a p$ are shown. Conservative substitutions are indicated by a plus sign $|+|$. Nonconserved residues are indicated by a period (.). The gaps were introduced to permit the alignment of the first and second LIM repeats. The spacing of some of the conserved cysteine residues differs between proteins and between repeats (Freyd ct al. 1990). The conserved residues at distinct places in the two LIM repeats may suggest that they differ slightly in structure. Alternatively, these residues might be important for organizing the two repeats into a larger structural unit. (TCTP) T-cell translocation protein 1 , product of the Ttg-l gene, identified in a $\mathrm{T}$-cell leukemia cell line (McGuire et al. 1989). (B) Sequence comparison of the homeo domains of the LIM family. Residues most conserved in Drosophila homeo domain proteins are indicated above the ap sequence. The tyrosine residue at position 25 is not conserved. ap has an asparagine residue at this position. The other LIM proteins have basic residues at this position. By virtue of their particular pattern of amino acid identities and conservative substitutions, the LIM proteins appear to define a new class of homeo domain. (C) Schematic representation of the organization of the LIM proteins, aligned with respect to the homeo domain. ap is the longest of the family at 469 -amino acid residues. In all cases, the LIM domains are adiacent and lie a variable distance to the amino-terminal side of the homeo domain. The locations of putative transcriptional activation domains are indicated. (PPPP' A proline-rich domain in $a p$ and lin-11; (QQQQ) A glutamine-rich domain in is 1,$1 ;---)$ an acidic domain in mec-3. The locations of the first residue of the LIM and homeo domains are indicated.

overlap in the region of the disc that is affected in the respective mutants.

$v g$ encodes a nuclear protein that lacks any known DNA-binding motif and that contains a histidine-rich domain that is thought to mediate protein-protein interaction (Williams et al. 1991). The LIM domain has also been suggested to mediate protein-protein interaction (Freyd et al. 1990). The similarity in the patterns of ap and $v g$ expression and in their mutant phenotypes raises the intriguing possibility that their protein product genes might act together as regulators of imaginal disc development. One set of experiments has suggested that these genes might be implicated in organization of the spatial pattern of the wing. Bownes and Roberts (1981) produced chimeric wing discs composed of wild-type and $v g$ mutant cells. In a chimeric disc, $v g$ mutant cells can form structures normally absent in the homozygous $\mathrm{vg}$ mutant disc, suggesting that the mutant cells are capable of responding to normal patterning cues provided by wild-type cells in the chimera. These observations sug- gest that the elaboration of the positional cues leading to development of the wing blade may be defective in the mutant discs while the ability of cells to respond to these cues is relatively normal (Bownes and Roberts 1981).

\section{How are the dorsal appendages specified?}

Snodgrass (1935) has suggested that wings arose during insect evolution as outgrowth from the dorsal thoracic body wall. This view implies the need for acquisition of new gene activities to promote the development of wing structures. At present, the nature of any such signals that discriminate presumptive wing from body wall remains unclear. In this context, it may be helpful to compare the genetic bases of wing and leg development. In the leg disc the distinction between leg and body wall is an active process. The homeo box gene Distal-less (DII) is expressed in a subset of leg imaginal disc cells and is required in these cells to promote the development of limb structures above the developmental "ground state" 
of the body wall (Cohen and Jürgens 1989; Cohen et al. 1989; B. Cohen, A.A. Simcox, and S.M. Cohen, in prep.). Both genetic mosaic analysis and embryo culture experiments show that in the absence of D11 activity, imaginal disc cells only form body wall structures/Cohen and Jürgens 1989; B. Cohen, A.A. Simcox, and S.M. Cohen, in prep.). It remains to be determined whether any of the genes required for wing development play a role analogous to $D I 1$. We can exclude $d d d$ as a candidate for this function because $d d d$ is required nonautonomously in the entire disc (Simcox et al. 1987). Although $a p$ and $v g$ mutants lack wing blade structures, both genes are expressed at some level in the region of the wing disc that gives rise to thoracic body wall structures. In this respect, they differ from Dll, which is not expressed in the presumptive ventral body wall. Because none of the known genes required for wing blade development appears to function precisely analogously to Dll, development of the wing and haltere appendages may follow different rules than development of the leg.

\section{Materials and methods}

ap genomic and cDNA clones

Genomic DNA flanking the P-element insertion in rK568 was cloned by plasmid rescue (Bier et al. 1989; Wilson et al. 1989). A 200-bp fragment was recovered in the plasmid and was used to screen a genomic library of Canton-S DNA cloned in $\lambda$ Dash II (provided by Ron Davis, Cold Spring Harbor Laboratory, Cold Spring Harbor, NY). Additional clones covering the ap regions were isolated from this library using an ap cDNA probe. cDNA clones were isolated from an imaginal disc cDNA library (provided by J. Botas). Seven cDNA clones representing two transcript size classes were characterized. Most clones are near full length, based on restriction mapping and DNA sequence of the 5 ' ends of several cDNAs. One cDNA (cAP-B) was fully sequenced on both strands. Partial sequence was derived from cDNAs cAP-A, -E, - F, - G, and - H. In addition, the locations of exons in the genomic DNA were determined by hybridization with cDNA probes, and the exons were sequenced to determine the detailed organization of the transcription unit. DNA sequencing was carried out with the help of the DNA sequence core facility in the Institute for Molecular Genetics, Baylor College of Medicine. The amino acid sequence of the deduced ap protein was used to screen PIR (release 30.0), SWISS-PROT /release 19.0), Gen Pept (release 69.0), and Gen Pept update (Dec 10 1991), using the BLAST network service at National Center for Biotechnology Information.

\section{ap $m R N A$ expression}

The pattern of expression of the ap transcript was determined by whole-mount in situ hybridization to embryos, essentially as described by Tautz and Pfeifle (1989). Whole-mount labeling of imaginal discs was performed as described previously (Phillips et al. 1990; Cohen et al. 1991). To examine the pattern of ap expression during the early stages of disc eversion, 1- to 6-hr-old prepupae were dissected from the pupal case and processed for staining as described for larval imaginal discs. The pattern of $\beta$-galactosidase activity driven by the rK568 enhancer detector insertion was visualized by histochemical staining as described by Bellen et al. (1989). Double-labeling procedures were carried out as described in Cohen et al. (1991).

The $a p^{r K 568}$ insertional mutant and the excision allele $a p^{U G O 35}$ were tested to determine whether the ap transcript was expressed in homozygous mutant animals. Embryos and imaginal discs from strains of the genotype ap/CyO, $\left.\mathrm{P} / \mathrm{ry}^{+}\right]$ $(w g / l a c Z)$ were double-labeled to visualize $\beta$-galactosidase activity and, subsequently, by in situ hybridization to visualize $a p$ transcript. Homozygous mutant individuals can be identified by the absence of $\beta$-galactosidase staining in the pattern of the wingless gene, indicating the absence of the $\mathrm{CyO}, \mathrm{P}_{\left[\mathrm{ry}^{+}\right]}$( $\mathrm{wg} /$ lacZ) balancer chromosome. $a p^{r K 568}$ homozygotes express the ap transcript; $a p^{U G O 35}$ homozygotes do not express the ap transcript in the embryo or the imaginal discs (data not shown).

\section{Drosophila strains and crosses}

A collection of enhancer detector insertion strains was provided by Ulrike Gaul, Linda Higgins, and Gerry Rubin (University of California, Berkeley). rK568 was identified on the basis of a homozygous wingless phenotype and subsequently shown to be an allele of $a p$. Two other P-element insertions from this screen, $\mathrm{rH} 7$ and rQ107, may also be inserted at the ap locus. These insertions produce the same pattern of $\beta$-galactosidase staining in embryos and imaginal discs as $\mathrm{rK} 568$; however, neither produces a mutant phenotype when homozygous. Like rK568, rQ107 maps to the cytogenetic interval 41F-42A. The identification of $\mathrm{rH} 7$ and $\mathrm{rQ107}$ as innocuous insertions in the ap locus is tentative, because DNA flanking these inserts has not been cloned.

A series of deletions of the nap gene was used to further define the location of the rK568 insertion. $D f(2 R)$ nap $^{9-2}, D f(2 R) n^{12} p^{12}$, and $D f(2 R) b w^{v D e 2 L} C y^{R}$ delimit the region containing ap to the cytogenetic region $41 \mathrm{~F} 8-42 \mathrm{~A} 1,2$ (R. Kreber, pers. comm.). This region contains the gene affected by the rK568 insertion.

Mobilization of the resident $P$ element was performed using a defective $\mathrm{P}$ element on the third chromosome, $\left.\mathrm{P} / \mathrm{ry}^{+} \Delta 2-3\right]$ (99B), which serves as a source of transposase but is, itself, virtually immobile (Robertson et al. 1988). A genetic screen to recover excisions is described in Figure 3 . Five hundred putative excisions of the rK568 insertion were identified as $r y^{-}$males at step 3. The males were backcrossed to lethal (2) $P\left(\mathrm{ry}^{+} / \mathrm{b}\right.$ Adh $\mathrm{cn} / \mathrm{CyO} ; \mathrm{ry}^{506}$ to allow stocks to be established from single excision events. A total of 175 stocks were examined. Of these, 96 lines produced wild-type progeny as homozygotes, suggesting that $55 \%$ of excisions reverted to wild type. One of these lines, UGO5, has been analyzed molecularly and found to produce an apparently precise excision (Fig. 3 |. The remaining $45 \%$ of excisions produced homozygous viable flies with ap mutant phenotypes. Of these, 41 of $175(23.4 \%)$ produced a strong ap phenotype like the original rK568 insertion, 18 of $175(10.2 \%)$ produced a less severely affected strap-like wing, 13 of 175 $(7.4 \%)$ produced a relatively normal wing with a defective hinge, causing the wing to be held out at an abnormal angle, and 7 of $175(4 \%)$ were pupal lethal. A subset of lines was pupal lethal. Because the null allele is viable, pupal lethality may reflect a disruption of genes in addition to ap.

\section{Acknowledgments}

We are very grateful to Ulrike Gaul, Linda Higgins, and Gerry Rubin for providing the enhancer detector lines, among which rK568 was found, and to Bob Kreber for providing nap deletions and other ap alleles. We thank Norbert Perrimon for the $\mathrm{CyO}$, 
$\left.P / \mathrm{ry}^{+}\right]$(wg/lacZ) chromosome and Hugo Bellen for the lethal $\{2\}$ $\mathrm{P}\left[\mathrm{ry}^{+}\right] \mathrm{b}$ Adh $\mathrm{cn} / \mathrm{CyO}$; ry ${ }^{506}$ stock. We thank Phil Soriano and Juan Botas for comments on the manuscript and Judi Cooper for secretarial assistance. This work was supported in part by the Howard Hughes Medical Institute and by Basil O'Connor Starter Scholar Research award 5-91-510 to S.C.

The publication costs of this article were defrayed in part by payment of page charges. This article must therefore be hereby marked "advertisement" in accordance with 18 USC section 1734 solely to indicate this fact.

\section{Note added in proof}

Sequence data described in this paper have been submitted to the EMBL/GenBank data libraries under accession no. X65158.

\section{References}

Altartz, M., S.W. Applebaum, D.S. Richard, L.I. Gilbert, and D. Segal. 1991. Regulation of juvenile hormone synthesis in wild type and ap mutant Drosophila. Mol. Cell. Endocrinol. 81: 205-216.

Baker, N.E. 1988. Embryonic and imaginal requirements for wingless, a segment-polarity gene in Drosophila. Dev. Biol. 125: 96-108.

Bate, M., E. Rushton, and D. Currie. 1991. Cells with persistent twist expression are the cmbryonic precursors of adult muscles in Drosophila. Development 113: 79-89.

Bellen, H., C.J. O'Kane, C. Wilson, U. Grossniklaus, R.K. Pearson, and W.J. Gehring. 1989. P element mediated enhancer detection: A versatile method to study development in Drosophila. Genes. (4) Dev. 3: 1288-1300.

Bier, E., H. Vaessin, S. Shepherd, K. Lee, K. McCall, S. Barbel, L. Ackerman, R. Carretto, T. Uemura, E. Grell, L.-Y. Jan, and Y.-N. Jan. 1989. Searching for pattern and mutation in the Drosophila genome with a P-lacZ vector. Genes \& Dev. 3: 1273-1287.

Bodmer, R. and Y.N. Jan. 1987. Morphological differentiation of the embryonic peripheral neurons in Drosophila. Wilhelm Roux's Arch. Dev. Biol. 196: 69-77.

Bownes, M. and S. Roberts. 1981. Regulative properties of wing discs from the vestigial mutant of Drosophila melanogaster. Differentiation 18: 89-96.

Butterworth, F.M. and R.C. King. 1965. The developmental genetics of ap mutants of Drosophila melanogaster. Genetics 52: $1153-1174$.

Bryant, P.J. 1978. Pattern formation in imaginal discs. In The genetics and biology of Drosophila ied. M. Ashburner and T.R.F. Wright), vol. 2c, pp. 229-235. Academic Press, New York.

Cavener, D. 1987. Comparison of the consensus sequence flanking translational start sites in Drosophila and vertebrates. Nucleic Acids Res. 15: 1353-1361.

Chalfie, M. and M. Au. 1989. Genetic control of differentiation of the Caenorhabditis elegans touch receptor neurons. Science 243: 1027-1033.

Cohen, B., E. Wimmer, and S.M. Cohen. 1991. Early development of the leg and wing primordia in the Drosophila embryo. Mech. Dev. 33: 229-240.

Cohen, S.M. and G. Jürgens. 1989. Proximal-distal pattern formation in Drosophila: Cell autonomous requirement for Distal-less gene activity in limb development. EMBO $\%$ 8: 2045-2055.

Cohen, S.M., G. Brönner, F. Küttner, G. Jürgens, and H. Jäckle. 1989. Distal-less encodes a homeodomain required for limb development in Drosophila. Nature 338: 432-434.

Dambly-Chaudiere, C. and A. Ghysen. 1987. Independent subpatterns of sense organs require independent genes of the achaete-scute complex in Drosophila larvae. Genes \& Dev. 1: 297-306.

Ferguson, E.L., P. Sternberg, and H.R. Horvitz. 1987. A genetic pathway for the specification of the vulval cell lineages of Caenorhabditis elegans. Nature 326: 259-267.

Freyd, G., S.K. Kim, and H.R. Horvitz. 1990. Novel cysteine-rich motif and homeodomain in the product of the Caenorhabditis elegans cell lineage gene lin-11. Nature 344: 876-879.

Fristrom, D. 1969. Cellular degeneration in the production of some mutant phenotypes in Drosophila melanogaster. Mol. Gen. Genet. 103: 363-379.

Ghysen, A., C. Dambly-Chaudiere, E. Aceves, L.Y. Jan, and Y.N Jan. 1986. Sensory neurons and peripheral pathways in Drosophila embryos. Wilhelm Roux's Arch. Dev. Biol. 195: 281-289.

Gonzalez, F., L. Swales, H. Bejsovic, H. Skaer, and A. MartinezArias, 1991. Secretion and movement of wingless protein in the epidermis of the Drosophila embryo. Mech. Dev. 35: 4354.

Ingham, P.W., A.M. Taylor, and Y. Nakano. 1991. Role of the Drosophila patched gene in positional signalling. Nature 353: 184-187.

Karlsson, O., S. Thor, T. Norberg, H. Ohlsson, and T. Edlund. 1990. Insulin gene enhancer binding protein Isl-1 is a member of a novel class of proteins containing both a homeodomain and a Cys-His domain. Nature 344: 879-882.

Kiss, I., G. Bencze, E. Fekete, A. Fodor, J. Gausz, P. Maroy, J. Szabad, and J. Szidonya. 1976. Isolation and characterization of X-linked lethal mutants affecting differentiation of the imaginal discs in Drosophila melanogaster. Theor. Appl. Genet. 48: 217-226.

Kornberg, T. 1981. engrailed: a gene controlling compartment and segment formation in Drosophila. Proc. Natl. Acad. Sci. 78: 1095-1099.

Li, P.M., J. Reichert, G. Freyd, H.R. Horvitz, and C.T. Walsh. 1991. The LIM region of a presumptive Caenorhabditis ele gans transcription factor is an iron-sulfur-and zinc-containing metallodomain. Proc. Natl. Acad. Sci. 88: 9210-9213.

Lindsley, D.L. and G. Zimm. 1985. Genes A-K. Dros. Inf. Serv. 62: 24-26.

McGuire, E.A., R.D. Hockett, K.M. Pollock, M.F. Bartholdi, S.J. O'Brien, and S.J. Korsmeyer. 1989. The $\mathrm{t}(11 ; 14)(\mathrm{pl} ; \mathrm{ql} 1)$ in a T-cell acute lymphoblastic leukaemia cell line activates multiple transcripts, including $\mathrm{Ttg}-1$, a gene encoding a potential zinc finger protein. Mol. Cell. Biol. 9: 2124-2132.

Mitchell, P.J. and R. Tiian. 1989. Transcriptional regulation in mammalian cells by sequence specific DNA binding proteins. Science 245: 371-378.

Padgett, R.W., R.D. St. Johnston, W.M. Gelbart. 1987. A transcript from a Drosophila pattern gene predicts a protein homologous to the transforming growth factor- $\beta$ family. $\mathrm{Na}$ ture 325: 81-84.

Phillips, R.G., I.J.H. Roberts, P.W. Ingham, and J.R.S. Whittle. 1990. The Drosophila segment polarity gene patched is involved in a position-signalling mechanism in imaginal discs. Development 110: 105-114.

Postlethwait, I.H. and A.M. Handler. 1978. Nonvitellogenic female sterile mutants and the regulation of vitellogenesis in Drosophila melanogaster. Dev. Biol. 67: 202-213.

Postlethwait, J.H. and K. Weiser. 1973. Vitellogenesis induced by juvenile hormone in the female sterile mutant ap-four in Drosophila melanogaster. Nature New Biol. 244: 284-285.

Robertson, H.M., C.R. Preston, R.W. Phillis, D.M. Johnson- 
Schlitz, W.K. Benz, and W.R. Engels. 1988. A stable genomic source of P-element transposase in Drosophila melanogaster. Genetics 118: 461-470.

Searles, L.L., A.L. Greanleaf, W.E. Kemp, and R.A. Voelker. 1986. Sites of P element insertion and structures of P element deletions in the $5^{\prime}$ region of Drosophila melanogaster RPII215. Mol. Cell. Biol. 6: 3312-3319.

Sharma, R.P. and V.L. Chopra. 1976. Effect of the wingless (wg') mutation on wing and haltere development in Drosophila melanogaster. Dev. Biol. 48: 461-465.

Shearn, A. 1978. Genetic control of imaginal disc development. In The genetics and biology of Drosophila (ed. M. Ashburner and T.R.F. Wright), vol. 2c, pp. 443-510. Academic Press, New York.

Shearn, A. and A. Garen. 1974. Genetic control of imaginal disc development in Drosophila. Proc. Natl. Acad. Sci. 71: 13931397.

Shearn, A., T. Rice, A. Garen, W. Gehring. 1971. Imaginal disc abnormalities in lethal mutants of Drosophila. Proc. Natl. Acad. Sci. 68: 2594-2598.

Simcox, A.A., G. Wurst, E. Hersperger, and A. Shearn. 1987. The defective dorsal discs gene of Drosophila is required for the growth of specific imaginal discs. Dev. Biol. 122: 559-567.

Simcox, A.A., I.J.H. Roberts, E. Hersperger, M.C. Gribbin, A. Shearn, and J.R.S. Whittle. 1989. Imaginal discs can be recovered from cultured embryos mutant for the segment polarity genes engrailed, naked and patched but not from wingless. Development 107: 715-722.

Spencer, F., M. Hoffman, and W.M. Gelbart. 1982. Decapentaplegic: A gene complex affecting morphogenesis in Drosophila melanogaster. Cell 28: 451-461.

Snodgrass R.E. 1935. Principles of insect morphogenesis. McGraw-Hill, New York/London, UK.

St. Johnston, D. and C. Nüsslein-Volhard. 1992. The origin of pattern and polarity in the Drosophila embryo. Cell 68: 201219.

Tautz, D. and C. Pfeifle. 1989. A non-radioactive in situ hybridization method for the localization of specific RNAs in Drosophila embryos reveals translational control of the segmentation gene hunchback. Chromosoma 98: 81-85.

van den Heuvel, M., R. Nusse, P. Johnston, and P.A. Lawrence. 1989. Distribution of the wingless gene product in Drosophila embryos: A protein involved in cell-cell communication. Cell 59: 739-749.

Way, M.C. and M. Chalfie. 1988. mec3, a homcobox-containing gene that specifies differentiation of touch receptors neurons in C. elegans. Cell 54: 5-16.

. 1989. The mec3 gene of Caenorhabditis elegans requires its own product for maintained expression and is expressed in three neuronal cell types. Genes \& Dev. 3: 1823 1833.

Way, M.C., L. Wang, J.-Q. Run, and A. Wang. 1991. The mec3 gene contains cis-acting elements mediating positive and negative regulation in cells produced by asymmetric cell division in Caenorhabditis elegans. Genes \& Dev. 5: 21992211

Whittle, J.R.S. 1990. Pattern formation in imaginal discs. Sem. Cell Biol. 1: 241-252.

Wieschaus, E. and W.J. Gehring. 1976b. Clonal analysis of primordial disc cells in the early embryo of Drosophila melanogaster. Dev. Biol. 50: 249-263.

Williams, J.A., J.B. Bell, and S.B. Carroll. 1991. Control of Drosophila wing and haltere development by the nuclear vestigial gene product. Genes \& Dev. 5: 2481-2495.

Wilkins, A. and D. Gubb. 1991. Pattern formation in the embryo and imaginal discs of Drosophila: What are the links? Dev.
Biol. 1455: 1-12.

Wilson, T.G. 1981a. Expression of phenotypes in a temperaturesensitive allele of the ap mutation in Drosophila melanogaster. Dev. Biol. 85: 425-433.

Wilson, T.G. 1981b. A mosaic analysis of the ap mutation in Drosophila melanogaster. Dev. Biol. 85, 434-445.

Wilson, C., R.K. Pearson, H.J. Bellen, C. O'Kane, U. Grossniklaus, and W.J. Gehring. 1989. P-element-mediated enhancer detection: An efficient method for isolating and characterizing developmentally regulated genes in Drosophila. Genes \& Dev. 3: 1301-1313. 


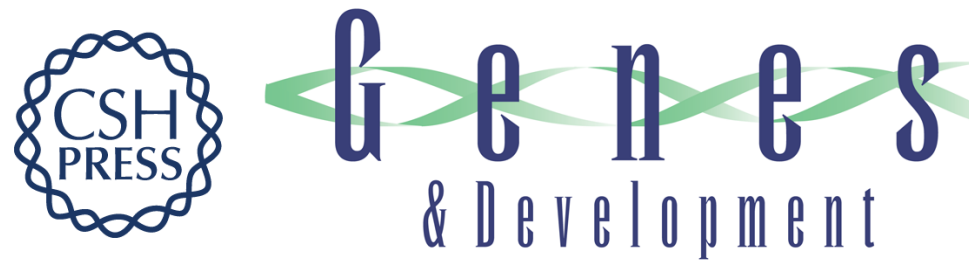

\section{apterous, a gene required for imaginal disc development in Drosophila encodes a member of the LIM family of developmental regulatory proteins.}

B Cohen, M E McGuffin, C Pfeifle, et al.

Genes Dev. 1992, 6:

Access the most recent version at doi:10.1101/gad.6.5.715

References This article cites 52 articles, 20 of which can be accessed free at: http://genesdev.cshlp.org/content/6/5/715.full.html\#ref-list-1

License

Email Alerting Service

Receive free email alerts when new articles cite this article - sign up in the box at the top right corner of the article or click here.

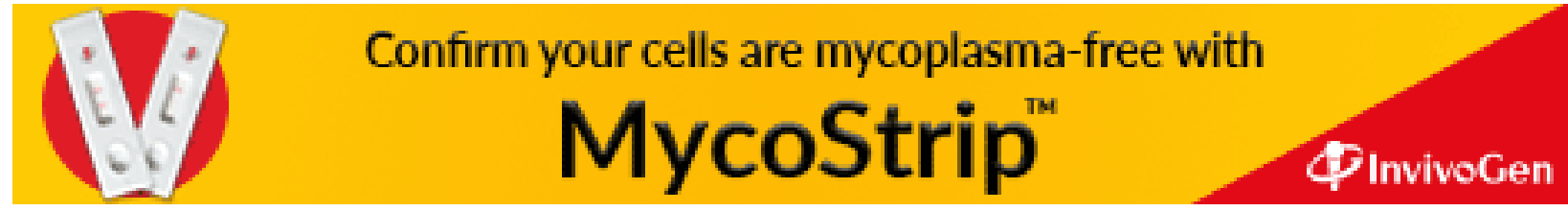

